\title{
What determines the visual persistence of complex stimuli?
}

\author{
JAMES G. MAY \\ University of New Orleans, Lakefront, New Orleans, Louisiana \\ and \\ ALYSIA B. RITTER \\ University of Houston, Houston, Texas
}

\begin{abstract}
Visual persistence has been shown to increase with the spatial frequency of sinusoidal and square-wave gratings. It has also been found to decrease with increasing contrast. If persistence is determined by visibility alone, then a square-wave grating containing greater power would be expected to give rise to less persistence than would a sinusoidal grating of the same fundamental spatial frequency. In the present experiment, measures of visual persistence were obtained with gratings composed of sinusoids, square waves, and square waves with the fundamental removed. Fundamental spatial frequency was varied from 0.5 to $4.5 \mathrm{cpd}$ in 1 -cpd steps. Visual persistence was found to increase only with the missing fundamental waveform. This suggests that visual persistence is determined by the lowest actual sinusoidal component of complex stimuli. Additional power contained in the higher harmonics of square-wave gratings does not serve to reduce persistence.
\end{abstract}

Visual persistence is defined as the subjective perseveration of a visual sensation beyond the objective termination of the physical stimulation for that sensation. Yet although this conceptualization appears straightforward, the measurement of this aspect of sensation is not trivial and has led to extensive investigation. Different techniques for the measurement of persistence have given rise to varied results (see Long, 1980), which has led to the suggestion that the perceptual phenomenon may be based on more than one physiological mechanism (Breitmeyer, Levi, \& Harwerth, 1981). One technique employed to estimate persistence involves determining the minimum interstimulus interval necessary to render a repetitively presented spatial stimulus perceptually continuous in time (Meyer \& Maguire, 1977). This procedure has been shown to result in differences in persistence when a number of stimulus variables are manipulated. Using this procedure, persistence has been found to: (1) decrease as a function of the contrast of the stimulus (Badcock \& Lovegrove, 1981); (2) increase as a function of the spatial frequency of the stimulus (Badcock \& Lovegrove, 1981; Meyer \& Maguire, 1977); (3) decrease as a function of stimulus duration (Badcock \& Lovegrove, 1981; Bowling \& Lovegrove, 1980); (4) be greater for horizontal and vertical as opposed to obliquely oriented gratings (Meyer \& L'Hommedieu, 1977; Schwartz, Winstead, \& May, 1982), and (5) decrease with adaptation to the stimulus (Meyer \& Maguire, 1981).

Correspondence should be addressed to James G. May, Department of Psychology, University of New Orleans, Lakefront, New Orleans, LA 70148.
In some of the studies cited above, unidimensional sinewave gratings were used, whereas in others, square-wave gratings or checkerboard stimuli were used. Although these stimuli vary in spatial complexity, all the relationships listed above can be obtained with each type. Sinewave gratings differ from square-wave gratings, in that the latter contain more spatial frequency components and greater net power. In the present experiment, we sought to determine whether complex waveforms, which contain more spatial frequency components and more net power, would persist more or less than simple waveforms with less net power. In addition, square-wave gratings with the fundamental component removed have the same global form as square-wave gratings, but contain fewer spatial components and less contrast. We asked whether stimuli with a "missing fundamental" component would give rise to more or less persistence than stimuli with all of the appropriate components.

Two experienced psychophysical observers served under all conditions. Both possessed natural or corrected $20 / 20$ visual acuity and were free from any other visual abnormalities. The stimuli were displayed on a small CRT (Hewlett-Packard 1332A-P31 phosphor). The screen was masked to produce a circular stimulus field (subtending $10^{\circ}$ of visual angle at $50 \mathrm{~cm}$ ), which was surrounded by an equiluminant field (subtending $40^{\circ}$ of visual angle) matched in chromaticity to that of the CRT display. The stimuli were provided by modulating the $z$ axis of the CRT with sine, square, and harmonic waveforms, which corresponded to gratings with fundamental spatial frequencies of $0.5,1.5,2.5,3.5$, and $4.5 \mathrm{cpd}$. Spatial profiles were provided by two function generators (Wavetek, 
Models 143 and 148), whose outputs were mixed and gated electronically. These spatial patterns were presented as a repetitive train of $40-\mathrm{msec}$ pulses, the interstimulus interval was varied in 10-msec steps, and each train consisted of 12 presentations of the stimulus. Under all conditions, the contrast of the sine wave was matched to the contrast of the fundamental sine-wave component in the square wave (i.e., the amplitude of the fundamental in the square wave was 1.4 times that of the pure sine wave). Missing fundamental waveforms were created by subtracting the fundamental sine-wave component from the square wave. Since the amplitude relationship among the three waveforms was fixed, the amplitude of the sine wave was used to determine contrast thresholds.

Contrast thresholds were determined for 40 -msec presentations of each sine wave, using the method of adjustment. The contrast of each waveform was then set $15 \%$ above the contrast threshold for sine waves at each spatial frequency. The subjects adjusted the interstimulus interval until the grating appeared to have temporal continuity. They were allowed to "bracket" their threshold to find the minimal interstimulus interval that yielded a perceptually continuous grating. Five such determinations were made for each type of waveform at each of the five spatial frequencies $(0.5,1.5,2.5,3.5$, and $4.5 \mathrm{cpd})$.

The data for the 2 subjects are presented in Figure 1. The mean of the interstimulus interval values is plotted as a function of fundamental spatial frequency for each waveform. Over the range of spatial frequencies tested, no change in visual persistence was noted for sine- or square-wave stimuli for either subject. This is not surprising, since in previous studies it has been reported that large increases in persistence usually occur at higher spatial frequencies (Badcock \& Lovegrove, 1981; Meyer \& Maguire, 1979). More importantly, no difference in persistence between sine- and square-wave stimuli was noted at any spatial frequency for either subject. This suggests that the subjective offset of complex gratings is determined

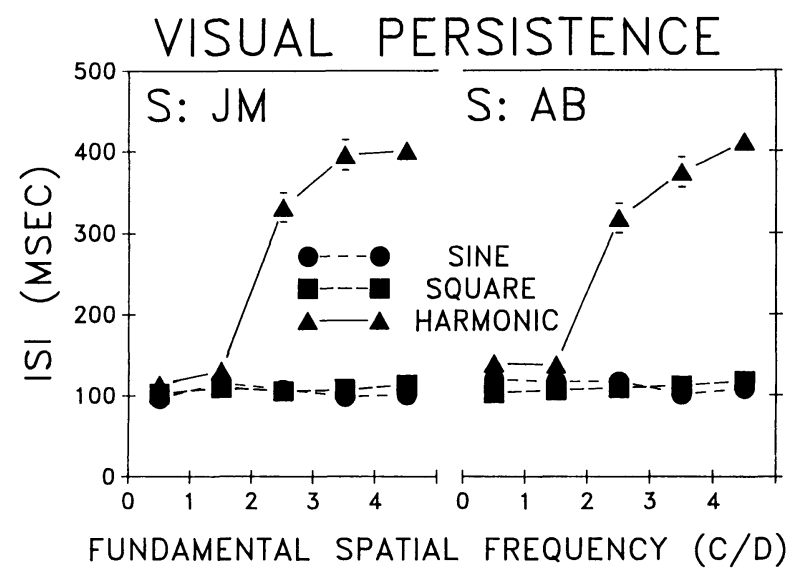

Figure 1. Mean visual persistence, for 2 subjects, as a function of fundamental spatial frequency for the three types of waveforms employed. by the most visible component. In these cases, that was the fundamental spatial frequency component. This view is supported when we consider the persistence observed with the harmonics waveform. Persistence increases abruptly when the (missing) fundamental frequency exceeds $1.5 \mathrm{cpd}$. This is understandable, since the third harmonic component is the most visible spatial component in this waveform. Increased persistence with spatial components above $7.5 \mathrm{cpd}$ (the third harmonic of the 2.5-cpd missing fundamental waveform) is well documented (Badcock \& Lovegrove, 1981; Meyer \& Maguire, 1979).

These results indicate that visual persistence is mediated by the most visible component in a complex grating and not by the complexity or net power of the waveform. Thus, as Bowling (1982) has reported, a square-wave grating, which has the same fundamental as a sine-wave grating, but contains higher frequency components and more net power, persists as long as a sine wave grating. And a missing fundamental waveform, which has the same harmonic composition as a square wave, but differs in fundamental content, persists significantly longer than a square wave. One explanation for why persistence increases under these conditions might be that the higher harmonics contain less contrast, which renders the stimulus less visible, but previous researchers (Marx \& May, 1982) have found that gratings of equal apparent contrast still give rise to increasing persistence with increased spatial frequency. Thus, differences in frequency cannot be accounted for merely on the basis of contrast sensitivity. However, Marx and May (1982) have shown that persistence remains constant across spatial frequency, if the duration of the stimulus has been adjusted to equate for the temporal integration differences between spatial frequency channels. In the present experiment, stimuli were equated for contrast sensitivity with regard to the fundamental components, but large differences in contrast sensitivity and temporal integration remained for the higher harmonic components. Since these components mediated visibility when the harmonic waveforms were used, it is reasonable to conclude that these factors underlie the differences noted.

This interpretation implies that the persistence of a complex stimulus can be predicted, given the knowledge of the spectral composition of the stimulus and the temporal integration properties of human spatial channels. It may well be that this conclusion holds only for complex stimuli of a periodic nature (i.e., gratings, checkerboards, etc.). Other complex stimuli, such as more natural images (e.g., faces, objects, landscapes), contain local differences in contrast and render a two-dimensional Fourier description of the stimulus less accurate. In those cases, persistence could be mediated by the features rather than by the more global aspects of the stimulus.

\section{REFERENCES}

BADCOCK, D., \& Lovegrove, W. (1981). The effects of contrast, stimulus duration, and spatial frequency on visible persistence in normal and specifically disabled readers. Journal of Experimental Psychology: Human Perception \& Performance, 7, 495-505. 
Bowling, A. (1982). Studies of human visible persistence. $\mathrm{PhD}$ thesis, University of Tasmania.

Bowling, A., \& Lovegrove, W. (1980). The effect of stimulus duration on the persistence of gratings. Perception \& Psychophysics, 27, 574-578.

BreitMeyer, B. G., LeVI, D. M., \& HARWERTH, R. S. (1981). Flicker masking in spatial vision. Vision Research, 21, 1377-1385.

LoNG, G. M. (1980). Iconic memory: A review and critique of the study of short-term visual storage. Psychological Bulletin, 88, 785-820.

MARX, M. S., \& MAY, J. G. (1982). The relationship between temporal integration and persistence. Vision Research, 23, 1101-1106.

MeYer, G. E., \& L'HommedieU, G. (1977). The oblique effect: Interactions with visual persistence and spatial configurations. Investigative Ophthalmology \& Visual Science (Suppl.), 123.
MeYer, G. E., \& MAGuiRe, W. M. (1977). Spatial frequency and the median of short-term visual storage. Science, 198, 524-525.

Meyer, G. E., \& Maguire, W. M. (1979). The effects of bar width and spatial frequency-specific adaptation on visual persistence. Bulletin of the Psychonomic Society, 14, 64-66.

MEYER, G. E., \& MAGUIRE, W. M. (1981). Effects of spatial-frequency specific adaptation and target duration on visual persistence. Journal of Experimental Psychology: Human Perception \& Performance,7, 151-156.

Schwartz, B., Winstead, D. K., \& May, J. G. (1982). Meridional differences in temporal resolution. Perception, 11, 25-35.

(Manuscript received June 23, 1989.) 